\title{
M-estimators as GMM for Stable Laws Discretizations
}

\author{
Davood Farbod \\ Quchan Institute of Engineering and Technology
}

\begin{abstract}
This paper is devoted to "Some Discrete Distributions Generated by Standard Stable Densities" (in short, Discrete Stable Densities). The large-sample properties of M-estimators as obtained by the "Generalized Method of Moments" (GMM) are discussed for such distributions. Some corollaries are proposed. Moreover, using the respective results we demonstrate the large-sample properties for a parametric function.
\end{abstract}

Keywords. Discrete stable densities; M-estimators as obtained by the GMM.

MSC 2010: 62F12, 62E20, 60E07.

\section{Introduction}

The Discrete Stable Densities have been suggested as a suitable model for applications in Bioinformatics (Astola and Danielian, 2007; Astola et al., 2007, 2010). One of the most important problems for Discrete Stable Densities is the statistical analysis for parameters estimators. But there exist essential difficulties for this distributions which are as follows:

(I) The second order moment (also for the most cases the first order moment) is infinite;

(II) There are not closed forms for probability mass functions;

(III) Until now, to the author's knowledge, there has not been proposed any closed form for generating and characteristic functions. 
These conclude that statistical inferences of parameters for such models are inappropriate. Statistical inferences for such discrete distributions have been studied by some authors (for example: Farbod and Gasparian, 2008a, 2008b; Farbod and Arzideh, 2011; Farbod, 2011). The sub-classes of maximally-skewed and symmetric Discrete Stable Densities are important in large-scale biomolecular systems. They can be suitable models to describe phenomena in large-scale biomolecular systems (Astola and Danielian, 2007, Ch. 2).

From (I) it follows that the methods based on moments are not applicable. The purpose of this paper is to demonstrate the validity of applying the $M$-Estimators as obtained by the GMM, under considering some special functions, for Discrete Stable Densities in maximally-skewed and symmetric cases.

The structure of the paper is as follows: In Section 2, we recall some preliminary concepts about Stable Distributions and Discrete Stable Densities. The main results of the paper are given in Section 3. A brief conclusion is provided in Section 4.

\section{Preliminaries}

The univariate stable distributions comprise the class of limiting distributions for normalized sums of independent and identically distributed random variables. The members of this class are described by four parameters: $\alpha \in(0,2]$ is the index or shape parameter, $\beta \in[-1,1]$ is the skewness parameter, $\gamma \in(0, \infty)$ is the scale parameter and $\delta \in(-\infty, \infty)$ is the location parameter. The parameter $\alpha$, is the main one, measures the degree of peakedness and the heaviness of the tails of the stable distribution. Parameter $\beta$ determines the amount and direction of skewness. For $\beta=1$ the distribution is maximally-skewed to the right, and is symmetric for $\beta=0$. For details on definitions and properties of stable distributions, the reader is referred to Astola and Danielian (2007); Firouzi and Mohammadpour (2009); Zolotarev (1986).

Supposing $s(x ; \alpha, \beta, \gamma, \delta)$ is a stable density and also $s(x ; \alpha, \beta)$ is standard stable density $(\gamma=1, \delta=0)$. Without loss of generality, in this paper the standard case is considered (Zolotarev, 1986, p. 60). Series expansions for densities of standard stable laws in literature are known. We have the following series expansions (Astola and Danielian, 2007): 
For $0<\alpha<1, \beta=1$ and $x>0$

$$
s(x ; \alpha, 1)=\frac{1}{\pi} \sum_{j=1}^{\infty} \frac{\Gamma(j \alpha+1)}{j !}(-1)^{j-1} x^{-\alpha j-1} \sin (\pi j \alpha) .
$$

The density function (1) is convergent as $0<\alpha<1$ and $x>0$. For details see Zolotarev (1986).

For $0<\alpha<1,1<\alpha<2, \beta=0$ and $x \geqslant 0$,

$$
s(x ; \alpha, 0)=\left\{\begin{array}{lll}
\frac{1}{\pi} \sum_{j=1}^{\infty} \frac{\Gamma(j \alpha+1)}{j !}(-1)^{j-1} x^{-\alpha j-1} \sin \left(\frac{\pi j \alpha}{2}\right), & x>0, & (a) \\
\frac{1}{\pi} \Gamma\left(1+\frac{1}{\alpha}\right) . & x=0 . & (b)
\end{array}\right.
$$

If $x<0$, instead of $s(x ; \alpha, 0)$ we use $s(-x ; \alpha, 0)$. It is well-known that for $0<\alpha<1$ the series $(2-a)$ is convergent for each $x \neq 0$, and for $1<\alpha<2$ may be justified as an asymptotic expansion when $x \longrightarrow \infty$. For details on this see Zolotarev (1986).

There are different ways for discretization of Stable Laws (Astola et al., 2010). In this paper we consider the discretization's way which is based on Stable Densities (for example: Farbod and Gasparian, 2008a, 2008b). Let us now have the following Discrete Stable Densities constructed from (1) and (2), respectively:

$$
p(x ; \alpha, 1)=\frac{s(x ; \alpha, 1)}{c_{\alpha, 1}}, \quad x=1,2, \ldots,
$$

where $c_{\alpha, 1}=\sum_{y=1}^{\infty} s(y ; \alpha, 1) ;$ and,

$$
p(x ; \alpha, 0)=\frac{s(x ; \alpha, 0)}{c_{\alpha, 0}}, \quad x=0,1,2, \ldots,
$$

where $c_{\alpha, 0}=\sum_{y=0}^{\infty} s(y ; \alpha, 0)$.

The contribution of this paper is to provide a discussion of asymptotic properties of $M$-estimators as obtained by the GMM for the models (3) and (4).

\section{M-estimators as obtained by the GMM}

Let us consider the following parametric space $(\gamma=1, \delta=0)$ :

$$
\Theta=\{(\alpha, \beta) \mid(0<\alpha<1, \beta=1),(0<\alpha<1 \text { or } 1<\alpha<2, \beta=0)\} .
$$


Assuming $X^{n}=\left(X_{1}, \ldots, X_{n}\right)$, with observations $x^{n}=\left(x_{1}, \ldots, x_{n}\right)$, is a sample from (3) or (4), and $D$ is some subset of $\Theta$ (for example, $\left.0<\alpha_{1} \leqslant \alpha \leqslant \alpha_{2}<1\right)$.

Supposing the function $g(x ; \alpha) \neq 0$ such that

$$
\tau(\alpha)=E_{\alpha}\left[g\left(X_{1} ; \alpha\right)\right]=0 .
$$

Definition (Borovkov, 1998). The value $\widehat{\alpha}$ which is a solution to the equation $\sum g\left(x_{i} ; \alpha\right)=0$ is called $M$-estimator as obtained by the GMM for the solution $\alpha$ to $\tau(\alpha)=0$.

We consider (Borovkov, 1998, p. 58)

$$
g(x ; \alpha)=g(x)-m(\alpha)
$$

where $m(\alpha)=E_{\alpha}\left[g\left(X_{1}\right)\right]$.

Note. If $g(x)=x^{r}, r \geqslant \alpha$ then for the models (3) and (4), $E_{\alpha}\left[X^{r}\right]=\infty$. So, in order to use M-Estimators as obtained by the GMM we need to consider $g(x)$ such that its mathematical expectation to be finite. Moreover, in the Mestimator method the function $m(\alpha)=E_{\alpha}\left[g\left(X_{1}\right)\right]$ should be monotone and continuous by $\alpha$ (Borovkov, 1998, p. 55). We have the following Lemmas.

Lemma 1. We have:

(i) For the model (3), $E_{\alpha}\left[g\left(X_{1}\right)\right]<\infty$ when $g(x)=\ln ^{k} x, k \in \mathbb{N}$ or $g(x)=x^{r}, r<\alpha$;

(ii) For the model (4), $E_{\alpha}\left[g\left(X_{1}\right)\right]<\infty$ when $g(x)=x^{r}, r<\alpha$.

The proof is obvious.

Lemma 2. The function $m(\alpha)=E_{\alpha}\left[g\left(X_{1}\right)\right]$ is strictly increasing and continuous with respect to $\alpha$ for:

(i) the model (3) when $g(x)=\ln ^{k} x, k \in \mathbb{N}$ or $g(x)=x^{r}, r<\alpha$;

(ii) the model (4) when $g(x)=x^{r}, r<\alpha$. 
Proof. (i) Let us assume $g(x)=\ln ^{k} x, k \in \mathbb{N}$. From definition of mathematical expectation it follows that

$$
m(\alpha)=\frac{\sum_{x=1}^{\infty} \ln ^{k} x s(x ; \alpha, 1)}{\sum_{y=1}^{\infty} s(y ; \alpha, 1)} .
$$

In order for $m(\alpha)$ to be strictly increasing it suffices to prove that $m^{\prime}(\alpha)=$ $\frac{\partial m(\alpha)}{\partial \alpha}>0$. This is equivalent to show that $A(\alpha)+B(\alpha)>0$ where

$$
\left\{\begin{array}{l}
A(\alpha)=\left\{\sum_{x=1}^{\infty} \ln ^{k} x s^{\prime}(x ; \alpha, 1)\right\}\left\{\sum_{y=1}^{\infty} s(y ; \alpha, 1)\right\}, s^{\prime}(x ; \alpha, 1)=\frac{\partial s(x ; \alpha, 1)}{\partial \alpha}, \\
B(\alpha)=-\left\{\sum_{x=1}^{\infty} \ln ^{k} x s(x ; \alpha, 1)\right\}\left\{\sum_{y=1}^{\infty} s^{\prime}(y ; \alpha, 1)\right\} .
\end{array}\right.
$$

We know that (Farbod and Gasparian, 2008b)

$$
\begin{aligned}
s^{\prime}(x ; \alpha, 1)= & \frac{1}{\pi} \sum_{j=1}^{\infty} \frac{\Gamma^{\prime}(j \alpha+1)}{(j-1) !}(-1)^{j-1} x^{-\alpha j-1} \sin (\pi j \alpha) \\
& +\frac{1}{\pi} \sum_{j=1}^{\infty} \frac{\Gamma(j \alpha+1)}{(j-1) !}(-1)^{j-1} x^{-\alpha j-1}\{\pi \cos (\pi j \alpha) \\
& -(\ln x) \sin (\pi j \alpha)\} .
\end{aligned}
$$

Substituting (1) and (6) into (5) and after some simplifications it concludes $A(\alpha)+B(\alpha)>0$ finishing the proof. The proof of continuity is obvious. The same proof is satisfied for the model (3) when $g(x)=x^{r}, r<\alpha$.

The proof of (ii) is met, similarly. The Lemma 2 is proved.

\subsection{Asymptotic Properties}

Case 1: for the model (3). We assume

$$
g(x)=\ln ^{k} x, \quad k \in \mathbb{N} \text { is fixed. }
$$

Let us have the following conditions, under which the strong consistency and asymptotic normality of the M-estimators as obtained by the GMM are satisfied. The conditions are (compare to Borovkov, 1998):

C1. The set $D \subset \Theta$ is compact. 
C2. We have

$$
\sup _{\alpha \in \Theta}|g(x ; \alpha)|<M(x),
$$

such that

$$
E_{\alpha}\left[M\left(X_{1}\right)\right]<\infty .
$$

C3. The function $g(x ; \alpha)$, for almost all $x$, is continuous in $\alpha$.

C4. The equation $\tau(\alpha)=0$ has a unique solution $\alpha_{0}$ in $D$ ( $\alpha_{0}$ is the initial value of $\alpha$ ).

C5. The function $g(x ; \alpha)$ is continuously differentiable in $\alpha$ for almost all $x$, and

$$
\sup _{\alpha \in D}\left|g^{\prime}(x ; \alpha)\right|<H(x), \quad g^{\prime}(x ; \alpha)=\frac{\partial g(x ; \alpha)}{\partial \alpha},
$$

such that $E_{\alpha}\left[H\left(X_{1}\right)\right]<\infty$.

C6. $\tau^{\prime}\left(\alpha_{0}\right) \neq 0$.

C7. $\sigma_{\alpha}^{2}=E_{\alpha}\left[g^{2}\left(X_{1} ; \alpha\right)\right]<\infty$.

Proposition 1. The conditions C1, C2,.., C7 are satisfied for the model (3).

Proof. The conditions C1, C3, C4 and C6 hold, obviously. It remains to demonstrate $\mathrm{C} 2, \mathrm{C} 5$ and $\mathrm{C} 7$.

Proof of C2. We have

$$
g(x ; \alpha)=g(x)-\sum_{x=1}^{\infty} g(x) p(x ; \alpha, 1)=\ln ^{k} x-\frac{\sum_{x=1}^{\infty} \ln ^{k} x s(x ; \alpha, 1)}{\sum_{y=1}^{\infty} s(y ; \alpha, 1)} .
$$

After some simplifications it follows that $M(x)=O\left(\ln ^{k} x\right), k \in \mathbb{N}$ is fixed.

It is enough to show that

$$
\sum_{x=1}^{\infty} \ln ^{k} x s(x ; \alpha, 1)<\infty
$$


which to be met by using Lemma 1 .

Proof of C5. Obviously, $g(x ; \alpha)$ is continuously differentiable at $\alpha \in D$. Taking derivative at $\alpha$ we have

$$
\begin{aligned}
g^{\prime}(x ; \alpha) & =-m^{\prime}(\alpha) \\
& =-\frac{\left(\sum_{x=1}^{\infty} \ln ^{k} x s^{\prime}(x ; \alpha, 1)\right)\left(\sum_{y=1}^{\infty} s(y ; \alpha, 1)\right)-\left(\sum_{x=1}^{\infty} \ln ^{k} x s(x ; \alpha, 1)\right)\left(\sum_{y=1}^{\infty} s^{\prime}(y ; \alpha, 1)\right)}{\left(\sum_{y=1}^{\infty} s(y ; \alpha, 1)\right)^{2}} .
\end{aligned}
$$

Substituting (1) and (6) into (7) and by some simplifications it follows that

$$
H(x)=O\left(\ln ^{k+1} x\right), \quad k \in \mathbb{N} \text { is fixed. }
$$

It remains to show that

$$
\sum_{x=1}^{\infty} \ln ^{k+1}(x) p(x ; \alpha, 1)<\infty,
$$

which again follows from Lemma 1 . The proof is finish.

Proof of C7. We have

$$
\begin{aligned}
\sigma_{\alpha}^{2} & =E\left[g^{2}\left(X_{1} ; \alpha\right)\right] \\
& =\frac{\sum_{x=1}^{\infty}\left(\ln ^{k} x-m(\alpha)\right)^{2} s(x ; \alpha, 1)}{\sum_{y=1}^{\infty} s(y ; \alpha, 1)} \\
& =\frac{\sum_{x=1}^{\infty} \ln ^{2 k} x s(x ; \alpha, 1)-2 m(\alpha) \sum_{x=1}^{\infty} \ln ^{k} x s(x ; \alpha, 1)+m^{2}(\alpha) \sum_{x=1}^{\infty} s(x ; \alpha, 1)}{\sum_{y=1}^{\infty} s(y ; \alpha, 1)} .
\end{aligned}
$$

We need to show that the denominator in (8) is finite, which concludes after setting (1) into (8).

The Proposition 1 is proved.

Corollary 1. The Proposition 1 holds when $g(x)=x^{r}, r<\alpha$, as well.

The proof is the same as Proposition 1.

Case 2: for the model (4). The same as Case 1 we have the following.

Proposition 2. The conditions C1, C2, .., C7 are met for the model 
(4) when $g(x)=x^{r}, \quad r<\alpha$.

The Proof is similar to Proposition 1.

Now, from the above respective results (Cases 1 and 2) for the models (3) and (4) we have:

Lemma 3. Assuming $X^{n}=\left(X_{1}, \ldots, X_{n}\right)$ is sample from (3) or (4). If conditions C1, C2 and C3 are satisfied, then as $n \longrightarrow \infty$ we have

$$
\sup _{\alpha \in D}\left|\tau_{n}^{\star}(\alpha)-\tau(\alpha)\right| \stackrel{\mathbb{P}, \text { a.s. }}{\longrightarrow} 0, \quad \text { (a.s. means almost suerly), }
$$

where $\tau_{n}^{\star}(\alpha)=\frac{1}{n} \sum_{i=1}^{n} g\left(x_{i} ; \alpha\right)$.

Proof. This follows from Borovkov (1998, Lemma 14.1, p. 62).

Theorem 1. Suppose that $X^{n}=\left(X_{1}, \ldots, X_{n}\right)$ is sample from (3) or (4). If the above mentioned conditions $C 1, C 2, \ldots, C 7$ are met, then the $M$-estimator as obtained by the $G M M, \widehat{\alpha}_{n}$, is:

a) strongly consistent, i.e.

$$
\widehat{\alpha}_{n} \stackrel{\mathbb{P}, \text { a.s. }}{\longrightarrow} \alpha
$$

b) asymptotically normal with coefficient $\sigma_{\alpha}^{2} \cdot\left[\tau^{\prime}(\alpha)\right]^{-2}$, i.e.

$$
\sqrt{n}\left(\widehat{\alpha}_{n}-\alpha\right) \stackrel{d}{\longrightarrow} N\left(0, \sigma_{\alpha}^{2} \cdot\left[\tau^{\prime}(\alpha)\right]^{-2}\right) .
$$

Proof. See Borovkov (1998, p. 62, p. 63).

In order to apply interval estimation of $\alpha$ we need to estimate $\sigma_{\alpha}^{2}\left[\tau^{\prime}(\alpha)\right]^{-2}$ which is not easy. To overcome this disadvantage we can consider the consistent estimator for $\sigma_{\alpha}^{2}\left[\tau^{\prime}(\alpha)\right]^{-2}$. Let us have the following.

Corollary 2. We have

$$
\widehat{\sigma_{\alpha}^{2}}\left[\tau^{\prime}\left(\widehat{\alpha}_{n}\right)\right]^{-2} \stackrel{\mathbb{P}}{\longrightarrow} \sigma_{\alpha}^{2}\left[\tau^{\prime}(\alpha)\right]^{-2} .
$$

Proof. Consider the model (3). It follows from the well-known continuity Theorem (Shiryaev, 1996) that

$$
g^{\prime}\left(x ; \widehat{\alpha}_{n}\right) \stackrel{\mathbb{P}}{\longrightarrow} g^{\prime}(x ; \alpha), \text { and } p\left(x ; \widehat{\alpha}_{n}, 1\right) \stackrel{\mathbb{P}}{\longrightarrow} p(x ; \alpha, 1) .
$$


It is readily seen that

$$
\left[\tau^{\prime}\left(\widehat{\alpha}_{n}\right)\right]^{-2} \stackrel{\mathbb{P}}{\longrightarrow}\left[\tau^{\prime}(\alpha)\right]^{-2}
$$

Also, we obtain

$$
\widehat{\sigma_{\alpha}^{2}} \stackrel{\mathbb{P}}{\longrightarrow} \sigma_{\alpha}^{2}
$$

From (9) and (10) the proof is complete. Similar proof holds for the model (4).

The proof is finished.

Corollary 3. Corollary 2 implies that interval estimation can be done for $\widehat{\alpha}_{n}$ when $n \longrightarrow \infty$.

\subsection{On A Parametric Function of the Index $\alpha$}

Let us here consider the model (3). Supposing

$$
U\left[s\left(X_{1} ; \alpha, 1\right)\right]=(\ln s(x ; \alpha, 1))^{\prime}=\frac{s^{\prime}(x ; \alpha, 1)}{s(x ; \alpha, 1)},
$$

is a contribution function from (1). We have the following.

Theorem 2. Assume that $\widehat{\alpha}_{n}$ is M-estimator as obtained by the GMM of the index $\alpha$ based on $g(x)$ for the model (3). The statistic

$$
\varphi_{n}^{\star}=\frac{1}{n I\left(\widehat{\alpha}_{n}\right)} \cdot U\left[s\left(X^{n} ; \widehat{\alpha}_{n}, 1\right)\right]
$$

is consistent, asymptotically unbiased and asymptotically normal of the parametric function

$$
\varphi(\alpha)=\frac{c_{\alpha, 1}^{\prime}}{c_{\alpha, 1} \cdot I(\alpha)}, \quad c_{\alpha, 1}^{\prime}=\frac{\partial c_{\alpha, 1}}{\partial \alpha} .
$$

Proof. In accord with continuity of the functions $I(\alpha)$ and $U[s(x ; \alpha, 1)]$ at $\alpha$ it concludes

$$
\begin{aligned}
\lim _{n \longrightarrow \infty} E_{\alpha}\left(\varphi_{n}^{\star}\right) & =\lim _{n \longrightarrow \infty} E_{\alpha}\left\{I^{-1}\left(\widehat{\alpha}_{n}\right) \cdot U\left[s\left(X^{n} ; \widehat{\alpha}_{n}, 1\right)\right]\right\} \\
& =\frac{c_{\alpha, 1}^{\prime}}{c_{\alpha, 1} \cdot I(\alpha)} \\
& =\varphi(\alpha),
\end{aligned}
$$


such that $\varphi_{n}^{\star}$ is asymptotically unbiased.

From (11) we have

$$
I(\alpha)=E_{\alpha}\left[U^{2}\left(p\left(X_{1} ; \alpha, 1\right)\right)\right]=\operatorname{var}_{\alpha}\left\{U\left[s\left(X_{1} ; \alpha, 1\right)\right]\right\} .
$$

It follows from (12) and (13) that $\varphi_{n}^{\star} \stackrel{\mathbb{P}}{\longrightarrow} \varphi(\alpha)$.

In addition, according to the Central Limit Theorem and the Delta Method (Oehlert, 1992) we conclude

$$
\sqrt{n}\left\{\varphi_{n}^{\star}-\varphi(\alpha)\right\} \stackrel{\mathbb{P}}{\longrightarrow} \eta \in N\left(0, \Omega_{\alpha}^{2} \cdot\left[\varphi^{\prime}(\alpha)\right]^{2}\right), \quad \Omega_{\alpha}^{2}=\sigma_{\alpha}^{2} \cdot\left[\tau^{\prime}(\alpha)\right]^{-2},
$$

which implies the estimator $\varphi_{n}^{\star}$ is asymptotically normal.

The proof is complete.

Corollary 4. Theorem 2 holds for the model (4) as well.

Corollary 5. We have

$$
\widehat{\Omega_{\alpha}^{2}} \cdot\left[\varphi^{\prime}\left(\widehat{\alpha}_{n}\right)\right]^{2} \stackrel{\mathbb{P}}{\longrightarrow} \Omega_{\alpha}^{2} \cdot\left[\varphi^{\prime}(\alpha)\right]^{2} .
$$

The proof is the same as Corollary 2 .

\section{Conclusion}

$M$-estimators as obtained by the GMM of the index parameter $\alpha$ have been studied for the models (3) and (4). We have proved strong consistency and asymptotic normality for them. To consider statistical inferences the consistent estimator has been proposed for $\sigma_{\alpha}^{2}\left[\tau^{\prime}(\alpha)\right]^{-2}$ as well. Then, the asymptotic properties have been proved for a parametric function of the index parameter $\alpha$ (Theorem 2).

\section{Acknowledgement}

The author would like to thank the two anonymous referees for their valuable comments. 


\section{References}

Astola, J. and Danielian, E. (2007). Frequency Distributions in Biomolecular Systems and Growing Network. Tampere International Center for Signal Processing (TICSP), series no. 31, Tampere, Finland.

Astola, J., Danielian, E. and Arakelyan, A.H. (2007). Frequency distributions in growing biomolecular networks based on stable densities. J. Reports National Academy Sciences Armenia, 107, 26-36.

Astola, J., Danielian, E. and Arzumanyan, S.K. (2010). Frequency distributions in bioinformatics: the development. A review, J. Proceedings Yerevan State University: Phys. Math. Sci., 223, 3-22.

Borovkov, A.A. (1998). Mathematical Statistics. Gordon and Breach Science Publishers, (translated from original Russian edition).

Farbod, D. and Gasparian, K.V. (2008a). Asymptotic properties of maximum likelihood estimator for some discrete distributions generated by Cauchy stable law. J. STATISTICA, 68, 321-326.

Farbod, D. and Gasparian, K.V. (2008b). The asymptotic properties of discrete distributions generated by standard skewed stable laws. Proceedings $9_{\text {th }}$ Iranian Statistical Conference, University of Isfahan, Isfahan, Iran, 138-144.

Farbod, D. and Arzideh, K. (2011). Asymptotic properties of moment estimators for distributions generated by Levy's Law. International Journal Applied Mathematics and Statistics, CESER Publications, 20(M11), 55-59.

Farbod, D. (2011). On the moment estimators for stable laws discretizations. Proceedings $8_{t h}$ Iranian Seminar on Probability and Stochastic Processes, University of Guilan, Rasht, Iran, 115-118.

Firouzi, M. and Mohammadpour, A. (2009). A survey on simulating stable random variables. Journal of Statistical Research of Iran, 6, 25-36, (in Persian).

Oehlert, G.W. (1992). A Note on the Delta Method, J. The American Statistician, 46, 27-29.

Shiryaev, A.N. (1996). Probability (Graduated Texts in Mathematics). Springer-verlag, 2nd edition, (translated from original Russian edition).

Zolotarev, V.M. (1986). One-dimensional Stable Distributions. American Mathematical Society, (translated from original 1983 Russian edition, Odnomernye Ustoichivye Raspredeleniya).

J. Statist. Res. Iran 8 (2011): 85-96 


\section{Davood Farbod}

Department of Mathematics,

Quchan Institute of Engineering and Technology,

Quchan, 94717-84686,

Quchan, Razavi Khorasan Province, Iran.

email: d.farbod@gmail.com

d.farbod@qiet.ac.ir 